\title{
RESEARCH HIGHLIGHT Can anti-obesity drugs be repurposed to treat cocaine addiction?
}

\author{
David J. Reiner ${ }^{1}$ and Jennifer M. Bossert ${ }^{1}$ \\ Neuropsychopharmacology (2018) 43:1983-1984; https://doi.org/10.1038/s41386-018-0035-7
}

\begin{abstract}
Cocaine is a potent psychostimulant and repeated cocaine use can lead to addiction and other adverse health consequences. According to the most recent results from the National Survey on Drug Use and Health, the percent of people aged 12 or older currently using cocaine has shown little change since 2007 (National Survey on Drug Use and Health, September 2017). This finding indicates that despite efforts over the past decade to decrease the number of people who use cocaine, its use continues to be a significant economic, social, and healthcare burden. Furthermore, the number of deaths from cocaine overdose has increased steadily between 2012 and 2015, and the number of cocaine deaths in 2015 was the second highest since 1999 (National Center for Health Statistics, CDC Wonder).

A major obstacle in the treatment of addiction to cocaine and other drugs of abuse is relapse to drug use after periods of abstinence. In former cocaine users, drug craving and relapse during abstinence are often triggered by cues and stimuli that were previously associated with cocaine use [1]. This clinical scenario is studied by using the reinstatement model in which laboratory animals are tested for reinstatement of drug seeking after exposure to different manipulations such as drug primes or drug cues previously paired with drug intake. The advantage of using such rodent models has been to test whether certain drugs or peptides will decrease drug-seeking behavior with the hope that success in the rodent model will eventually translate into human clinical trials. This is especially important for cocaine abuse since there are currently no efficacious clinically approved treatments.
\end{abstract}

Neuropeptides that are classically associated with the control of food intake can also modulate drug self-administration and reinstatement of drug seeking [2]. One such neuropeptide is glucagon-like peptide-1 (GLP-1), which is produced peripherally by the intestines and centrally by preproglucagon (PPG) neurons in nucleus tractus solitarius (NTS). Endogenous GLP-1 reduces food intake and body weight and promotes insulin release, and exogenous GLP-1 receptor (GLP-1R) agonists are clinically approved for treating type II diabetes mellitus and obesity [3]. More recently, studies in rodents found that GLP-1R agonists decrease cocaine self-administration and conditioned place preference [4]. In this issue of Neuropsychopharmacology, Hernandez et al. expand on these findings by testing the effects of GLP-1 using the reinstatement model of drug relapse. The authors first found that systemic administration of the GLP-1R agonist, exendin-4, decreases both cocaine- and cue-induced reinstatement of cocaine seeking at doses that do not reduce food intake or body weight, and do not produce nausea/malaise or motor deficits that can impair lever pressing. They also showed that peripheral administration of fluorescently tagged exendin-4 crosses the blood-brain barrier and acts on astrocytes and dopamine neurons in ventral tegmental area (VTA). Next, Hernandez et al. showed that VTA injections of exendin-4 decrease cocaine priming-induced reinstatement of cocaine seeking. Additionally, VTA injections of the GLP-1R antagonist exendin-(9-39) reverse the effects of systemically administered exendin- 4 on reinstatement of cocaine seeking. Lastly, the authors found a significant decrease in PPG mRNA in the NTS after rats' lever pressing was extinguished for 7 days, suggesting that extinction training reduces endogenous GLP-1 expression in the brain.

These findings, which extend previous work by the Schmidt and Hayes groups on the role of central GLP-1Rs in cocaine selfadministration $[5,6]$, are of potential translational significance. Systemically administered GLP-1R agonists decrease both drug priming- and cue-induced reinstatement of cocaine seeking at doses that do not affect food intake, body weight, and nausea/ malaise. GLP-1-based drugs (exenatide and liraglutide) are already clinically approved for the treatment of type II diabetes mellitus and obesity. Therefore, it is possible that these GLP-1R agonists could be repurposed to treat cocaine use, and as the present findings indicate cocaine relapse. Indeed, preliminary clinical research on the interaction between cocaine use and endogenous GLP-1 found that intravenous cocaine decreases plasma GLP-1 concentration and that plasma GLP-1 concentration was associated with the subjective response to cocaine [7].

Second, Hernandez et al. showed that VTA GLP-1Rs mediate reinstatement of cocaine seeking. These data provide an important foundation for the mechanistic understanding of the regional and cell-type-specific effects (VTA dopamine neurons and astrocytes) of GLP-1 on cocaine seeking. Importantly, central GLP$1 \mathrm{R}$ activation decreases cocaine-evoked phasic dopamine release in nucleus accumbens core [8], suggesting that VTA GLP-1R activation may dampen VTA dopamine cell firing, following cocaine exposure. The authors are well suited to follow up on their findings with mechanistic studies exploring the role of specific GLP-1R-expressing VTA neuronal subtypes in mediating cocaine seeking. Furthermore, the finding that systemically delivered fluorescent exendin-4 acts on VTA astrocytes supports a growing body of evidence that astrocytes express GLP-1R and take up systemically delivered GLP-1R agonists $[6,9]$, and provides additional support of the hypothesis that astrocytes play a role in reinstatement of cocaine seeking [10]. Manipulating astrocytes using pharmacological or chemogenetic techniques is a logical

\footnotetext{
${ }^{1}$ Behavioral Neuroscience Branch, IRP/NIDA/NIH, 251 Bayview Blvd, Suite 200, 08A721, Baltimore, MD 21224, USA

Correspondence: David J. Reiner (david.reiner@nih.gov) or Jennifer M. Bossert (jennifer.bossert@nih.gov)
}

Received: 30 January 2018 Revised: 9 February 2018 Accepted: 16 February 2018

Published online: 17 April 2018 
1984

next step in determining a functional role for GLP-1R-expressing VTA astrocytes in mediating cocaine seeking.

Finally, the authors connect GLP-1R VTA-mediated reinstatement of cocaine seeking to decreased PPG expression following 7 days of extinction but observed no change in VTA GLP-1R expression during extinction. These data provide complementary evidence of an endogenous GLP-1 mechanism, suggesting that a decrease in PPG expression following extinction may result in decreased GLP-1 release in downstream projection targets (e.g., VTA), which thereby promotes cocaine seeking upon exposure to drug priming or cues. Systemic or local injection of exendin-4 in VTA effectively restores VTA GLP-1 tone and rescues this effect. Using dual virus approaches to either knockdown PPG expression in VTA-projecting NTS PPG neurons or to inhibit these neurons would allow the authors to directly test this hypothesis. However, the underlying mechanisms that result in the downregulation of PPG expression should also be investigated and may have implications for the role of central GLP-1 expression in the development and maintenance of diabetes and obesity. In addition, as NTS PPG neurons project widely throughout the central nervous system [3], other sites expressing GLP-1R may play a role in cocaine seeking. Indeed, this group recently published data showing that GLP-1Rs in nucleus accumbens also play a role in reinstatement of cocaine seeking [6].

Collectively, Hernandez et al. extend the growing body of literature on the role of GLP-1 on natural reward-related behaviors to animal models of cocaine self-administration and relapse. The authors also provide preclinical evidence for GLP-1-based drugs that are already clinically approved for the treatment of diabetes and obesity to be potentially repurposed for cocaine addiction. Given preclinical and clinical work on the role of GLP-1 in alcohol intake [11], the current findings highlight the GLP-1 system as an important potential therapeutic target across drug classes. The present data will hopefully inspire future preclinical work on the role of GLP-1-based drugs in motivated behaviors and assist in the translation to clinical trials focused on GLP-1 as a potential treatment for addiction to cocaine and other drugs of abuse, including alcohol.

\section{ACKNOWLEDGEMENTS}

We thank Dr. Yavin Shaham for his insightful comments of this manuscript.
FUNDING:

Supported by the Intramural Research Program of NIDA (Yavin Shaham).

\section{ADDITIONAL INFORMATION}

Competing interests: The authors declare no competing interests.

Publisher's note: Springer Nature remains neutral with regard to jurisdictional claims in published maps and institutional affiliations.

\section{REFERENCES}

1. O'Brien CP, Childress AR, McLellan AT, Ehrman R. Classical conditioning in drugdependent humans. Ann N Y Acad Sci. 1992;654:400-415.

2. DiLeone RJ, Taylor JR, Picciotto MR. The drive to eat: comparisons and distinctions between mechanisms of food reward and drug addiction. Nat Neurosci. 2012;15:1330-1335.

3. Kanoski SE, Hayes MR, Skibicka KP. GLP-1 and weight loss: unraveling the diverse neural circuitry. Am J Physiol Regul Integr Comp Physiol. 2016;310:R885-895.

4. Hayes MR, Schmidt HD. GLP-1 influences food and drug reward. Curr Opin Behav Sci. 2016;9:66-70.

5. Schmidt HD, Mietlicki-Baase EG, Ige KY, Maurer JJ, Reiner DJ, Zimmer DJ, et al. Glucagon-like peptide-1 receptor activation in the ventral tegmental area decreases the reinforcing efficacy of cocaine. Neuropsychopharmacology. 2016;41:1917-1928.

6. Hernandez NS, O'Donovan B, Ortinski PI, Schmidt HD. Activation of glucagon-like peptide- 1 receptors in the nucleus accumbens attenuates cocaine seeking in rats. Addict Biol. 2017 [epub ahead of print].PMID: 2922661711.

7. Bouhlal S, Ellefsen KN, Sheskier MB, Singley E, Pirard S, Gorelick DA, et al. Acute effects of intravenous cocaine administration on serum concentrations of ghrelin, amylin, glucagon-like peptide-1, insulin, leptin and peptide $Y Y$ and relationships with cardiorespiratory and subjective responses. Drug Alcohol Depend. 2017;180:68-75

8. Fortin SM, Roitman MF. Central GLP-1 receptor activation modulates cocaineevoked phasic dopamine signaling in the nucleus accumbens core. Physiol Behav. 2017;176:17-25.

9. Reiner DJ, Mietlicki-Baase EG, McGrath LE, Zimmer DJ, Bence KK, Sousa GL, et al. Astrocytes regulate GLP-1 receptor-mediated effects on energy balance. J Neurosci. 2016;36:3531-3540.

10. Scofield MD, Boger HA, Smith RJ, Li H, Haydon PG, Kalivas PW. Gq-DREADD selectively initiates glial glutamate release and inhibits cue-induced cocaine seeking. Biol Psychiatry. 2015;78:441-451.

11. Jerlhag E. GLP-1 signaling and alcohol-mediated behaviors: preclinical and clinical evidence. Neuropharmacology. 2018 [epub ahead of print]. PMID: 29337226. 